\title{
Discovery of a $<210>$-Fiber Texture in Medical-Grade Metastable Beta Titanium Wire
}

Song Cai*, Jeremy E. Schaffer, Yang Ren, Mark R. Daymond

Song Cai*, Ph.D., J. E. Schaffer, Ph.D.

${ }^{1}$ Fort Wayne Metals Research Products Corporation, 9609 Ardmore Ave., Fort Wayne, IN 46809

USA

song_cai@fwmetals.com

Y. Ren, Ph.D.

${ }^{2}$ Advanced Photon Source, Argonne National Laboratory, 9700 S. Cass Ave, 433/D008, Argonne, IL 60439 USA

Prof. M. R. Daymond

${ }^{3}$ Dept. of Mech. and Mater. Eng., Queen's University, Nicol Hall, 60 Union Street, Kingston, Ontario, K7L 3N6, Canada

Keywords: beta titanium, 332 twinning, polycrystal plasticity modeling, synchrotron x-ray diffraction, wire texture

\section{ABSTRACT}

Texture and phase evolution of metastable $\beta$-III Ti alloy wires, produced in a medical-grade wire processing facility, are examined via synchrotron X-ray diffraction. The texture development in the $\beta$-phase was interpreted by a simple visco-plastic self-consistent (VPSC) modeling approach. Both the stress-induced martensite (SIM) and stress-induced omega (SIO) phase transformations are observed during the early stage of cold-deformation. The $\langle 110\rangle_{\beta}$ texture is gradually replaced by the $\left\langle 210>_{\beta}\right.$ texture at cold work levels above $50 \%$ total area reduction or equivalently 0.70 axial true strain. Formation of the $\langle 210\rangle_{\beta}$-fiber from the combined activity of $\{112\}$ and $\{332\}$ twinning plus conventional slip is observed and may not directly depend upon the stress-induced phase per se. According to the VPSC model, similar texture should occur in other metastable $\beta$-Ti alloys subjected to similar wire processing. These data should help inform process-structure-function towards better wire design in titanium-based medical devices. 


\section{Introduction}

$\beta$-Ti alloys have been utilized in the medical device industry at an increasing rate for their proven biological performance and relatively high elastic strain compared to $\alpha$ and $\alpha+\beta \mathrm{Ti}$ alloys. This favorable combination of properties arises because of a relatively compliant elastic modulus, high strength, and with specific processing, inelastic recoverable strain from the stressinduced phase transformation. ${ }^{[1,2]}$ For applications like intravascular guidewires, a large recoverable strain permits deployment through the complex vascular system to the distal target without permanent deformation. An elastic guidewire path helps mitigate tissue damage risk and provides enhanced proximal-to-distal torque control for accurate navigation and therapeutic delivery. ${ }^{[3]}$ These beneficial properties are highly texture, and therefore, process-dependent. In the $b c c$ crystal structure, the $\beta$-Ti alloy has the lowest modulus in the $<100>$ crystal direction, but high yield stress for dislocation slip in the $<111>$ direction due to low Schmid factors. ${ }^{[4]}$ The elastic strain associated with the Stress-induced Martensite (SIM) phase transformation is the largest along the $<110>$ direction. ${ }^{[5]}$ Therefore, understanding the deformation behavior and texture evolution during wire manufacture is critical for developing new materials, controlling processes within suitable bounds, and improving material properties for the next generation of Ti-based medical devices. Indeed, numerous studies have been carried out to investigate the deformation mechanisms of $\beta$-Ti alloys. ${ }^{[5-13]}$

\section{Deformation and Texture in Beta Titanium}

It is well known that deformation systems in $\beta$-Ti alloys include dislocation slip in the $<111>$ direction, $\{112\}$ and $\{332\}$ deformation twinning, ${ }^{[6-9]}$ and the stress-induced martensite (SIM) and stress-induced omega (SIO) phase transformation. ${ }^{[5,7,8,10,11]}$ The SIM phase ( $\alpha$ ") has an 
orthorhombic crystal structure; the SIO phase has an hcp crystal structure. As illustrated in Figure 1, the undistorted axes in the $\beta$-phase and the SIM are related as: $[100]_{\beta} \rightarrow[100]_{\alpha^{\prime \prime}}$ $[011]_{\beta} \rightarrow[010]_{\alpha^{\prime \prime}}$ and $[01 \overline{1}]_{\beta} \rightarrow[001]_{\alpha^{\prime \prime}}$. The orientation between the $\beta$ and the SIO axes follows: $\{111\}_{\beta} \rightarrow\{0001\}_{\omega}$ and $[1 \overline{1} 0]_{\beta} \rightarrow[11 \overline{2} 0]_{\omega}$. The SIM phase transformation causes expansion in one of the $<110>_{\beta}$ directions and contraction in one of the $<100>_{\beta}$ directions, where the energetically favorable variant depends on relationship between the crystal orientation and the loading direction. On the other hand, the SIO phase transformation causes small contractions in both of the $<111>_{\beta}$ and $<211>_{\beta}$ directions. ${ }^{[14]}$ The dominant systems which are active during deformation depend on many factors including: the stress-strain status, the phase stability and local and overall texture.

The evolution of texture and its influence on the mechanical properties of cold-rolled alloys have been extensively studied, ${ }^{[5,11-13]}$ the texture of $\beta$-Ti alloys after axisymmetrical deformation, such as cold drawing, has been largely ignored. There is still very little published research on the texture of cold-drawn $\beta$-Ti alloys. For materials with a body-centered cubic $(b c c)$ crystal structure, such as $\mathrm{V}, \mathrm{Nb}, \mathrm{Ta}, \mathrm{Mo}, \mathrm{W}$ and $\mathrm{Fe}$, the texture after cold drawing is the well-known $<110>_{\beta}$ fiber texture ${ }^{[15]}$ a similar texture would therefore be expected in cold-drawn $\beta$-Ti alloys. However, this is not the case in our recent studies, ${ }^{[16,17]}$ where the strong $\left.<210\right\rangle_{\beta}$ fiber texture rather than $<110>_{\beta}$ texture was observed in heavily cold-drawn $\beta$-III and Ti-15Mo alloys. In this paper, the evolution of texture and stress-induced phase transformation in a metastable $\beta$-Ti alloy during the cold-drawing process was tracked by using synchrotron X-ray diffraction on samples that were collected at selected processing steps. Mechanisms for the $\langle 210\rangle_{\beta}$ fiber texture are suggested via interpretation of a visco-plastic self-consistent (VPSC) model ${ }^{[18]}$ that has been 
successfully used in modeling textures of materials with different crystal structures under various stress-strain conditions. ${ }^{[19-22]}$

\section{Materials and Experiments}

Cold-drawn $\beta$-III titanium alloy wire comprising nominally $11 \mathrm{Mo}-5.8 \mathrm{Zr}-4.44 \mathrm{Sn}-0.14 \mathrm{O}$ (wt.\%) with balance titanium, commonly used in medical devices such as orthodontic arch wires, was processed for this study. Initial $2.5 \mathrm{~mm}$ wire stock was $\beta$-annealed at $815^{\circ} \mathrm{C}$ for 300 seconds in an inert argon atmosphere and cooled by flowing argon gas. After annealing, it was reduced in diameter by $85 \%$ using successive, multi-die, cold drawing (1.9 true strain) to a final diameter of $1 \mathrm{~mm}$. Samples were collected at selected points during the process. The texture of each sample was measured on the beam line 11-ID-C at the Advanced Photon Source (APS) at Argonne National Laboratory. A monochromatic X-ray beam with a wavelength of $0.10801 \AA$ was used with aperture-selected beam size of $0.5 \times 0.5 \mathrm{~mm}^{2}$. The experimental setup was similar to that in Ref. ${ }^{[23]}$. With this sample orientation, reflections from planes with normal oriented parallel to the sample axial direction are located at $90^{\circ}$ and $270^{\circ}$ on the Debye-Scherrer rings and were recorded on a two-dimensional (2-D) detector placed at approx. $1.8 \mathrm{~m}$ behind the sample. Due to the cylindrical sample symmetry, and assumed axisymmetric texture, only one diffraction image per sample sample was needed for texture analysis. Spiral or concentric textures associated with non-uniform surface to core deformation during wire drawing were not analyzed in this work due to beam versus sample size limitations. The data processing and texture analysis were carried out in FIT2D ${ }^{[24]}$ and MAUD ${ }^{[25]}$ software. First, each 2-D image was sliced ('caked') and integrated at a $5^{\circ}$ azimuth angle (i.e. from -2.5 to $2.5^{\circ}, 2.5$ to $7.5^{\circ}$, etc.) to produce 72 diffraction

spectra in FIT2D. These 72 spectra were then fitted by Rietveld refinement ${ }^{[26]}$ in MAUD to 
generate orientation distribution functions and to reconstruct pole figures. Textures and the stress-induced phase transformation were interpreted based on an overall goodness-of-fit via the least-squares-regression intrinsic to MAUD. Overall goodness-of-fit ranged from 9 to $10.5 \%$ for this experiment with less than $0.5 \%$ error in phase analysis.

\section{Experimental Results and Discussion}

Figure 2 shows a subset of diffraction image data that were taken throughout the cold drawing process ranging from $0 \%$ cold work $(\mathrm{CW}), 0$ true strain (ts) or annealed (Figure $2 \mathrm{a})$ to $50 \% \mathrm{CW}$, 0.70 ts (Figure 2b) and $85 \% \mathrm{CW}, 1.9$ ts (Figure 2c). Figure 2a illustrates the moderate $\{110\}_{\beta}$ intensity present in the annealed sample at the 12 o'clock position corresponding to the axial direction. The intensity of the $\{110\}_{\beta}$ reflections in the axial direction was 3.12 multiples of random density (mrd), an indicator of a moderate fiber texture in the $\beta$-annealed state prior to cold deformation. The axial fiber intensity of the $\{110\}_{\beta}$ plane was maintained up to approximately $60 \% \mathrm{CW}, 0.92$ ts. Figure $2 \mathrm{~b}$ shows extra diffraction rings observed after early deformation (black arrows). At the higher cold work level, the strong $\{110\}_{\beta}$ intensity in the axial direction started to split and rotate towards the transverse direction, and eventually stabilized at approximately $10^{\circ}$ around the axial direction as evidenced by splitting in recorded intensity of the $\{110\}_{\beta} /\{220\}_{\beta}$ Debye-Scherrer rings of Figure 2c.

The appearance of additional diffraction rings shown in Figure $2 \mathrm{~b}$ suggest progress of stressinduced phase transformation, while the increasingly patterned diffraction intensities correspond to the development of texture during the cold drawing process. Both phenomena can be characterized by interpreting the 2-D diffraction image data by Rietveld refinement in the 
MAUD software. It is instructive to mention that, for the cold-drawn samples up to $50 \% \mathrm{CW}$, best visual and numerical fit were only obtained when both stress-induced martensite and stressinduced omega phases were included in the VPSC model. This is not surprising since both deformation modes have been reported for this alloy and because both should occur and exhaust early during heavy deformation. ${ }^{[5,7,8,10,11]}$ Figure $\mathbf{3}$ shows a comparison between the experimental and the modeling results and provides visual evidence of an accurate fit.

Figure 4 shows the evolution of the stress-induced phase transformation during the cold drawing process. It was evident that the majority of the stress-induced phases were produced during the early stage of the cold drawing process. For example, about $11 \%$ SIO and $22 \%$ SIM phases were formed by the first 20\% CW (0.22 ts), and another approx. 5\% SIO and 10\% SIM phases were produced by increasing the cold work to $50 \%$ ( 0.70 ts); after that, the stress-induced phase appeared to reach saturation and remain constant through $85 \% \mathrm{CW}$ (1.9 ts).

Figure 5 shows the evolution of the textures of the $\beta$-phase and the stress-induced phases during the cold drawing process. It was observed that the original $<110>_{\beta}$-fiber gradually shifted towards the $\langle 210\rangle_{\beta}$ direction at the beginning of cold drawing eventually achieving a strong $<210\rangle_{\beta}$-fiber after $85 \% \mathrm{CW}$. For the $\omega$-phase, a relatively strong $\langle 11 \overline{2} 0\rangle_{\omega}$ texture and relatively weak $\langle 10 \overline{1} 1\rangle_{\omega}$ and $\langle 11 \overline{2} 2\rangle_{\omega}$ textures were produced at the early stage of cold drawing. With increasing cold work, the strength of the $\langle 11 \overline{2} 2\rangle_{\omega}$ texture increased at the expense of the $\langle 11 \overline{2} 0\rangle_{\omega}$ and $\langle 10 \overline{1} 1\rangle_{\omega}$ textures eventually resulting in a strong $\langle 11 \overline{2} 2\rangle_{\omega^{-}}$ fiber $(7.5 \mathrm{mrd})$ after $85 \% \mathrm{CW}$. Fewer changes are observed in the inverse pole figure of the SIM phase. After $20 \% \mathrm{CW}\left(0.22\right.$ ts), a strong $\langle 041\rangle_{\alpha^{\prime}}$ and $\langle 014\rangle_{\alpha^{\prime}}$, texture were produced. The 
strength of the $\langle 041\rangle_{\alpha^{\prime \prime}}$, texture increased, while the strength of the $\langle 014\rangle_{\alpha^{\prime}}$, texture appears to be reduced by the continuous cold drawing.

Figure 6 shows selected $\beta$-phase pole figures to further inform the evolution of textures during cold wire drawing. With reference to the leftmost column of Figure 6, observe that the $\{110\}_{\beta}$ intensity remained concentrated in the wire axial direction up to $50 \% \mathrm{CW}$ (0.69 ts), beyond which it moved out and reappeared at $10^{\circ}$ around the axial direction. For both of the $\{100\}_{\beta}$ and $\{111\}_{\beta}$ planes, note that their intensities disposed around the axial direction increased by cold working. For the $\{100\}_{\beta}$ planes, an extra intensity ring was formed at approx. $15^{\circ}$ to the transverse direction after $85 \% \mathrm{CW}\left(1.9\right.$ ts). Transverse $\{111\}_{\beta}$ intensity moved slightly towards the axial direction, and concentrated at approx. $10^{\circ}$ from the transverse direction beyond about $75 \% \mathrm{CW}(1.4$ ts).

Figure 7 shows selected recalculated stress-induced phase pole figures from the sample with 85\% CW (1.9 ts). The orientation correspondence between the $\beta$-phase, SIO and SIM phases can be qualitatively discerned by comparing Figures 6 and 7. For example, the pole figure of the $\{0001\}_{\omega}$ plane shows a similar pattern to that of the $\{111\}_{\beta}$ plane. The $\{11 \overline{2} 0\}_{\omega},\{020\}_{\alpha^{\prime \prime}}$ and $\{002\}_{\alpha^{\prime \prime}}$ planes show a similar pattern to that of the $\{110\}_{\beta}$ plane. The $\{200\}_{\alpha^{\prime \prime}}$ plane exhibits the same intensity pattern as that of the $\{200\}_{\beta}$ plane. This is note surprising considering that the majority of the stress-induced phases were produced during the early stage of cold drawing process ( $<50 \% \mathrm{CW}, 0.70 \mathrm{ts})$. Subsequent plastic deformation up to $85 \% \mathrm{CW}$ (1.9 ts) would have destroyed the interphase orientation relationships. It is possible that the deformation in the $\omega$ phase was limited due to its nanoscale grain size ${ }^{[27-29]}$ Lacking significant slip, the $\omega$-phase may have simply rotated with the $\beta$-phase matrix during deformation, and therefore maintained the $\beta$ - 
phase-driven orientation relationship. Figure 5 shows that, with some intensity shift, the SIM texture components were maintained throughout the drawing process. This may evidence $<041>_{\alpha}$, texture as the stable SIM texture for the cold wire drawing process. These data could explain why the orientation relationship between the $\beta$-phase and the SIM phase that was produced after $20 \% \mathrm{CW}$ (0.22 ts) was still observed after $85 \% \mathrm{CW}$ (1.9 ts). A more rigorous description will require fully informed deformation modes and mechanisms in the SIO and SIM phases, which are unfortunately not available at this moment.

The aim this project was to understand the formation of the newly observed $<210>_{\beta}$ texture in cold-drawn $\beta$ Ti alloys and the impact of the stress-induced phase transformations SIO and SIM during deformation. Lack of detailed knowledge on the stress-induced phase transformations and the deformation mechanisms of the SIO and SIM phases after formation made this a difficult aim. Simplification of inherent complexity may be possible based on several observations from the current work, viz.: Figure 4 and 6 show that the stress-induced phase transformation is mostly active during the early stage of cold drawing $\left(<50 \% \mathrm{CW}, 0.70\right.$ ts); while the $<210>_{\beta}$ texture was produced after $50 \% \mathrm{CW}(0.70 \mathrm{ts})$. These results suggest that the formation of the $<210>_{\beta}$ texture may not be directly affected by the stress-induced phase transformation and is only caused by the activities of the dislocation slip and deformation twinning. Secondly, in spite of load-partitioning to the secondary SIO and SIM phases beyond 20\% CW (0.22 ts), and an altered $\beta$-phase load status, the macroscopic plastic flow experienced by the $\beta$-phase should be similar to that experienced by homogeneous $\beta$-phase due to the cylindrical sample geometry. In other words, the $\beta$-phase will maintain its cylindrical geometry during and after deformation because the constraint from the secondary phases is homogenous in the Poisson (transverse) direction. Therefore, even with the presence of secondary phases, the $\beta$-phase still can be treated 
macroscopically as a single phase material under approximately uniaxial tension. Based on this theory, a VPSC wire model comprising homogeneous $\beta$-phase was constructed and evaluated to simulate the formation of the $<210>_{\beta}$ texture during the cold drawing process.

\section{Modeling and Discussion}

In the VPSC model, a polycrystalline material is represented by weighted orientations (i.e. grains with different volume fractions). Each grain is treated as an ellipsoidal visco-plastic inclusion embedded in a homogeneous effective medium (HEM), which has the average properties of the polycrystal aggregate. The interaction between the individual grains and the HEM is treated by Eshelby tensor. ${ }^{[30]}$ The coupling between the stress deviations and the associated strain-rate deviations is represented by a parameter $n^{e f f}$. The effective compliance of the matrix increases as $n^{\text {eff }}$ increases. This parameter was set at 10 in the calculations, representing a compromise between the rigid upper-bound approximation and softer approximations such as the tangent selfconsistent model. ${ }^{[21]}$ The hardening of individual deformation systems is described by an extended Voce hardening law: ${ }^{[31]}$

$$
\tau^{s}=\tau_{0}^{s}+\left(\tau_{1}^{s}+\theta_{0}^{s} \Gamma\right)\left(1-\exp \left(-\theta_{0}^{s} \Gamma / \tau_{1}^{s}\right)\right)
$$

where $\tau_{s}$ is the instantaneous threshold value (i.e. critical resolved shear stress), $\tau_{0}^{s}$ is the Voce stress where the hardening extrapolates to zero, $\theta_{0}^{s}$ is the initial hardening rate, and $\tau_{1}^{s}$ is the final asymptotic hardening rate for the slip system $S$, and $\Gamma$ is the accumulated shear strain in the grain.

The effect of deformation twinning on texture is described by the predominant twin reorientation (PTR) scheme. ${ }^{[20]}$ Within each grain, the shear strain contributed by each twinning 
system and the associated volume fraction is monitored. The sum over all twin systems of a certain kind and over all grains represents the accumulated twin fraction in the aggregate. At each strain increment, the grains are fully re-orientated by the predominant twinning system (i.e. the one that has the highest accumulated volume fraction) provided certain conditions are fulfilled. More details about the VPSC model can be found in Ref. ${ }^{[18,22]}$.

The initial texture was generated by deforming a material with $\mathrm{N}=1000$ randomly orientated grains by the true tensile strain of 0.20 . Only the $\{110\}<111>$ slip system was activated to reproduce the weak $\langle 110\rangle_{\beta}$ texture observed in the annealed wire. This material was then tensile deformed to the true strain of 1.9 , equivalent to $85 \% \mathrm{CW}$. Modeled textures at selected points were used to compare with those shown in Figure 6. In order to re-produce the formation of the $<210>_{\beta}$ texture during the cold drawing process, the follow deformation systems were used: $\{110\}<111>$ dislocation slip, $\{112\}<111>$ and $\{332\}<113>$ twinning systems. While the characteristics of the $\{110\}$ slip and $\{112\}$ twining systems are well understood, the nature of the $\{332\}$ twinning system requires more discussion, especially concerning its shear direction and resultant shear strain. The widely accepted $\{332\}<113>$ twinning mode, illustrated in Figure 8, was proposed by R.H. Richman. ${ }^{[32]}$ In this model, referring to Figure 8, all atoms first shear along the $<113>$ direction to create a multiple lattice, half of these then shuffle perpendicular to the twinning plane to restore the original lattice such that the atom at the position B moves to position $\mathrm{C}$ and the atom at position B' moves to position B" after twinning. The shear strain is only approx. 0.35 , which is half of that for the $\{112\}$ twinning mode. The small shear strain of this twinning mode makes it energetically favorable. This model does not describe where the twin-plane-adjacent atoms (e.g. atoms A and D) go after twinning. Regardless of shuffle, it appears that these atoms will not be able to find energetically favorable sites in the re-orientated 
lattice. Current observations and successful modeling are based on an opposite direction

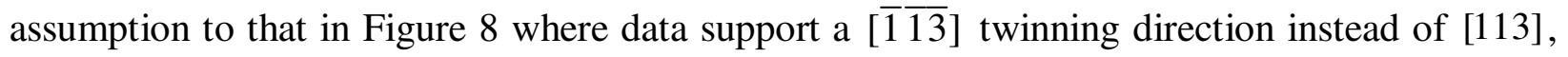
such that the atom at position $\mathrm{C}$ moves from position $\mathrm{A}$ instead of position $\mathrm{B}$. The shear strain for this move is admittedly high at 3.5 compared to 0.35 . The twelve $\{332\}<113>$ twinning modes used in this study are (233)[3 $\overline{1} \overline{1}],(\overline{22} 3)[\overline{3} 1 \overline{1}],(\overline{2} 33)[\overline{3} \overline{1} \overline{1}],(2 \overline{3} 3)[31 \overline{1}],(\overline{32} 3)[1 \overline{3} \overline{1}]$, (323)[ $[\overline{1} 3 \overline{1}],(3 \overline{2} 3)[\overline{1} \overline{3} \overline{1}],(\overline{3} 23)[13 \overline{1}],(3 \overline{3} 2)[\overline{1} 13],(332)[\overline{1} \overline{1} 3],(\overline{3} \overline{3} 2)[113]$, and $(\overline{3} 32)[1 \overline{1} 3]$. Note that these twinning systems have opposite twinning directions to those used by others. ${ }^{[3,34]}$ Based on previous observations, ${ }^{[27]}$ re-orientation by secondary twinning was allowed. With the latent hardening having been assumed to be equal to the self-hardening, the CRSS and Voce hardening parameters that give the overall best match are listed in Table 1 . Since only the texture was of interest and parameters were qualitatively fitted by VPSC modeling, these parameters represent arbitrary values and do not necessarily represent the actual strengths of different systems. The parameter ratios were considered of greater import since these define the relative activities of different deformation systems and thus control the texture development.

Figure 9 shows modeled pole figures of the $\{110\}_{\beta},\{100\}_{\beta}$ and $\{111\}_{\beta}$ planes at different cold work levels. It can be seen that, despite the simplicity of this modeling approach, all features presented in Figure 6 were nicely reproduced. For example, the VPSC model captured the $\{110\}_{\beta}$ intensity gradually moving out from the center axial direction at strains above $50 \%$ during the cold drawing process. The changes in the texture strength of the $\{200\}_{\beta}$ and $\{111\}_{\beta}$ planes around the axial and transverse sample directions were also well predicted. These data support our previous discussion that the activities of slip and twinning systems are responsible for formation of the cold-drawn $<210\rangle_{\beta}$-fiber texture. 
Figure 10 gives the relative activities calculated by the model for different deformation systems. According to the model, and in agreement with empirical diffraction data, dislocation slip maintained a relatively stable activity, while the activities of the $\{112\}$ and $\{332\}$ twinning systems showed opposite trends with cold work where they were equivalent at a true strain of approx. $0.80(55 \% \mathrm{CW})$. To understand the effect of each twinning mode on the texture, simulations were carried out on the same material with separate activation of each twinning system. These results show that the $\{112\}$ twinning produced a $<110>_{\beta}$ fiber texture, which is consistent with discussion that the orientation changes caused by the $\{112\}$ twinning should be the same as that caused by $\{110\}<111>$ dislocation slip. ${ }^{[35]}$ The $\{332\}$ twinning itself resulted in strong $<100>_{\beta}$ and $<111>_{\beta}$ textures. It was only through the combined activity of $\{112\}$ and $\{332\}$ twinning that $<210>_{\beta}$ texture was produced in the VPSC cold drawn wire model. Modeled dislocation slip slowed the rate of texture change, that is; without the $<111>$ dislocation slip the $<210>_{\beta}$ texture formed at true strains of less than 0.50 (approximately $40 \% \mathrm{CW}$ ).

The cold-drawn $<210>_{\beta}$ texture was reproduced by the VPSC model without considering the SIM and SIO phase transformation, yet stress-induced phases should be important components of texture evolution in early stages of wire reduction. In accordance with present diffraction data, early plastic deformation was dominated by the stress-induced phase transformation. For $20 \% \mathrm{CW}(0.22 \mathrm{ts})$, the model predicted an increased $<110>_{\beta}$ texture strength in the axial direction when not accounting for the stress-induced phase transformation. In this case, the SIM phase transformation will reduce the $\left\langle 110>_{\beta}\right.$ intensity but increase the $[010]_{\alpha}$, intensity in the tensile direction based on their orientation relationship.

The cylindrical sample geometry played an important role in the success of this simple modeling approach, where the cold drawing stress-strain distributions were assumed to be 
qualitatively unchanged by the SIM and SIO phases. The effect of load-partitioning caused by the secondary phases was then accommodated in fitting the modeling parameters. For samples with non-axisymmetric geometry, the constraint from the secondary phases would not be homogenous in the transverse directions and the assumed stress-strain status would be unlikely to generate a successful model. Ignoring secondary phases may produce significant errors. This may be one reason that S. Mercier et al. observed poor correlation using the $\{332\}$ twinning in modeling the cold rolled texture of a Ti20V alloy. ${ }^{[34]}$ A complete modeling approach should involve all of the secondary phases along with well-informed deformation mechanisms that were unavailable or beyond the scope of the current work and deserving of future attention.

Studies by S. Hanada and his colleagues demonstrated that the $\{332\}<113>$ twinning is closely related to the stability of the $\beta$-phase ${ }^{[9,36]}$ and that it only takes place in unstable $\beta$-Ti alloys. ${ }^{[37]}$ Thus, the $<210>_{\beta}$ texture should be observed in other metastable $\beta$-Ti alloys but not in stabilized $\beta$-Ti alloys. To confirm this and towards corroboration of our results, texture evolution in medical-grade wires comprising Ti-15Mo (Ti-14.4Mo-0.16O) and $\beta \mathrm{C}$ (Ti-4.17Mo3.78Zr-3.47Al-8.18V-5.88Cr-0.09O) alloys during cold drawing were also investigated. The former is a metastable $\beta$ alloy and the latter is a heavily stabilized $\beta$ alloy. ${ }^{[38]}$ Figure $\mathbf{1 1}$ shows the diffraction image and the inverse pole figure of the $\beta \mathrm{C}$ alloy with $94 \% \mathrm{CW}$. The stabilized $\beta$ C alloy exhibited the classic $<110\rangle_{\beta}$ fiber texture after $94 \% \mathrm{CW}$, while the Ti- 15 Mo alloy developed the same $\left\langle 210>_{\beta}\right.$ texture as that shown in Figure 1 and 5. These data corroborate our earlier results and suggest the absence of significant $\{332\}$ twinning activity in the stabilized- $\beta$ alloy. From these data we conclude that the newly reported $\langle 210\rangle_{\beta}$ texture found in cold-drawn $\beta$ III and Ti -15 Mo alloys is caused by the $\{332\}<113>$ twinning due to their low $\beta$-phase stability. 


\section{Conclusions}

The evolution of texture and phase transformation of the metastable $\beta$ III Ti alloy during the cold drawing process was studied by using synchrotron X-ray diffraction. The texture development in the $\beta$-phase is interpreted by a simple VPSC modeling approach. The following conclusions are made:

1. Both the SIM and SIO phase transformations are active, but mostly during the early stage of the cold drawing process.

2. The $\langle 110\rangle_{\beta}$ texture is gradually replaced by the $\langle 210\rangle_{\beta}$ texture in the $\beta$-phase at cold work levels above $50 \%$.

3. The formation of the $\langle 210\rangle_{\beta}$ texture is the result of the combined activity of the $\{112\}$ and $\{332\}$ twinning activities, and may not be directly related to the stress-induced phase transformation. Similar texture should be observed in other cold-drawn metastable $\beta$-Ti alloys.

4. Texture data and VPSC modeling results suggest a reverse sense $\{332\}$ twinning

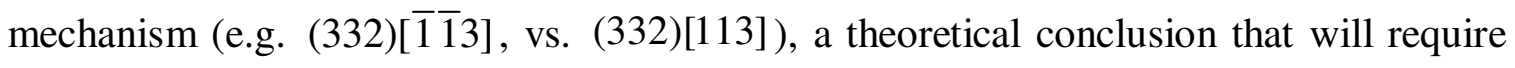
detailed TEM validation. 


\section{Acknowledgements}

X-ray diffraction experiments were carried out on beamline 11-ID-C at the APS at Argonne National Laboratory. Data analysis was performed using FIT2D and MAUD software. The VPSC modeling code was provided by C. Tomé. S.C. thanks Professor M.R. Daymond for instructive discussions, C. Yu (Ph.D. student) for X-ray diffraction and P. Sims for sample production. Funding and support from Fort Wayne Metals towards this project is acknowledged and greatly appreciated.

\section{References}

[1] M. Geetha, A.K. Singh, R. Asokamani, A.K. Gogia, Prog. Mater. Sci. 2009, 54, $397-425$.

[2] A.J. Goldberg and C.J. Burstone, J. Dent Res. 1979, 58, 593-599.

[3] S. Nuss, (Minnesota Medical Development, Inc.), US Patent 7,468,045, 2008.

[4] W. F. Hosford, Mechanical Behavior of Materials, Cambridge University Press, $2^{\text {nd }}$ edition, USA 2009.

[5] H.Y. Kim, T. Sasaki, K. Okutsu, J.I. Kim, T. Inamura, H. Hosoda, S. Miyazaki, Acta. Mater. 2006, 54, 423-433.

[6] S. Ishiyama, S. Hanada and O. Izumi, ISIJ International, 1991, 31, 807-813.

[7] H. Xing and J. Sun, Appl. Phys. Lett. 2008, 93, 031908.

[8] J.A. Roberson, S. Fujishiro, V.S. Arunachalam and C.M. Sargent, Metall. Trans. 1974, 5 2317-2322.

[9] S. Hanada and O. Izumi, Metall. Trans. 1980, 11A, 1447-1452.

[10] X. Zhao, M. Niinomi, M. Nakai, J. Hieda, Acta. Biomater. 2012, 8, 1990-1997.

[11] R.J. Talling, R.J. Dashwood, M. Jackson, D. Dye, Acta Mater. 2009, 57, 1188-1198.

[12] T. Inamura, Y. Kinoshita, J.I. Kim, H.Y. Kim, H. Hosoda, K. Wakashima, S.

Miyazaki, Mater. Sci. Eng. 2006, 438, 865-869. 
[13] S.J. Li, T.C. Cui, Y.L. Hao, R. Yang, Acta Biomater. 2008, 4, 305-317.

[14] S. K. Sikka, Y. K. Vohra and R. Chidambaram, Prog. Mater. Sci. 1982, 27, 245-310.

[15] H. Hu, Texture 1, 1974, 233-258.

[16] S. Cai, Y. Ren, L.E. Kay, Scripta. Mater. 2013, 68, 518-521.

[17] S. Cai, M.R. Daymond, Y. Ren, D.M. Bailey, L.E. Kay, Mater. Sci. Eng. A, 2013, $562,172-179$.

[18] R.A. Lebensohn, C. Tomé, Acta. Metall. Mater. 1993, 41, 2611-2624.

[19] I.J. Beyerlein, R.A. Lebensohn, C.N. Tomé, Mater. Sci. Eng. 2003, A345, 122-138.

[20] C.N. Tomé, R. A. Lebensohn and U.F. Kocks, Acta. Metall. Mater. 1991, 39, 26672680.

[21] F. Xu, R.A. Holt, M.R. Daymond, J. Nucl. Mater. 2009, 394, 9-19.

[22] C.N. Tomé and R. A. Lebensohn, Manual for Code Visco-Plastic Self-Consistent (VPSC), Version 7d, Los Alamos National Laboratory, USA 2012.

[23] H.-R. Wenk, S. Grigull, J. Appl. Cryst. 2003, 36, 1040-1049.

[24] A.P. Hammersley, FIT2-D V9.129 reference manual V3.1, ESRF internal report, USA 1998.

[25] L. Lutterotti, S. Matthies, H.-R. Wenk, A.S. Schulz, J.W. Richardson Jr., J. Appl. Phys. 1997, 81, 594-600.

[26] H. M. Rietveld, J. Appl Cryst. 1969, 2, 65-71.

[27] T. Furuhara, K. Kishimoto and T. Maki, Mater. Trans. JIM, 1994, 35, 843-850.

[28] X. Zhao, M. Niinomi, M. Nakai, G. Miyamoto, T. Furuhara, Acta. Biomater. 2011, 7, $3230-3236$.

[29] J.E. Schaffer, J. Mat. Eng. Perf. 2009, 18, 582-587. 
[30] J.D. Eshelby, Proc. R. Soc. Lond. 1957, 241, 376-396.

[31] C.N. Tomé, G.R. Canova, U.F. Kocks, N. Christodoulou, J.J. Jonas, Acta Metall. 1984, 32, 1637-1653.

[32] R.H. Richman, Deformation Twinning, R.E. Reed-Hill, J.P. Hirth, and H. C. Rogers, eds., Gordon and Breach, New York, NY, USA 1964, 237-271.

[33] E. Bertrand, P. Castany, I. Peron and T. Gloriant, Scripta Mater. 2011, 64, 11101113.

[34] S. Mercier, L.S. Tóth, A. Molinari, Text. Microstruct. 1995, 25, 45-61.

[35] F.W. Ling, E.A. Starke JR., B.G. Lefevre, Metall. Trans. 1974, 5, 179-187.

[36] S. Hanada and C. Izumi, Metall. Trans. 1987, 18A, 265-271.

[37] S. Hanada, M. Ozeki and O. Izumi, Metall. Trans. 1985, 16A, 789-795.

[38] G. Lütjuring and J.C. Williams, Titanium, Springer-Verlag, Berling Heidelberg, Germany 2003. 


\section{Figure Captions}

Figure 1 Schematic shows (a) the lattice relationship between the $\beta$ and omega phase, and (b) between the $\beta$ and stress induced martensite.

Figure 2 Diffraction image of a) beta III after anneal, b) beta III after 50\%CW (0.70 true strain) and c) beta III after $85 \% \mathrm{CW}$ (1.9 true strain). Vertical from the beam center is the sample axial direction. Arrows in e) show the diffractions of the stress induced phases.

Figure 3 Comparison between the experimental results (lower) and the fitted results (upper), beta III Ti, 85\% CW (1.9 true strain).

Figure 4 Evolution of SIO and SIM phases in beta III Ti alloy during cold drawing process. Each data point is the average of three samples.

Figure 5 Inverse pole figures from measured and fit data of $\beta$-phase, SIO and SIM phases in beta III Ti alloy during cold drawing process. Cold-work given for each image row in percent reduction and accumulated true strain (TS).

Figure 6 Pole figures from measured and fit data of $\beta$-phase in beta III Ti alloy during cold drawing process. Associated planes are given at the top of each column and the center pole is the sample axial direction. Cold-work is given for each image row in percent reduction and accumulated true strain (TS).

Figure 7 Selected pole figures of the SIO (upper) and the SIM (lower) in the beta III Ti sample with $85 \%$ cold work (1.9 accumulated axial true strain). Center pole is the sample axial direction.

Figure 8 (1 $1 \overline{1} 0)$ projection of the $(33 \overline{2})$ [113] twinning. Solid symbols represent atoms in the projection plane; Open symbols are atoms on planes at 0.707 a above or below the projection plane. Small dots in the upper area illustrate the original atom positions before twinning [31].

Figure 9 Modeled texture of $\beta$-phase during cold drawing. Center is the sample axial direction. Cold-work given for each image row in percent reduction and accumulated true strain (TS).

Figure 10 Relative activities of different deformation systems in the model through true tensile strain of about 1.8 , similar to about $85 \%$ wire cold work reduction. 
Figure 11 Diffraction image a) and inverse pole figure b) of the beta $\mathrm{C}$ after $94 \% \mathrm{CW}$ (2.8 true strain). 
(a)

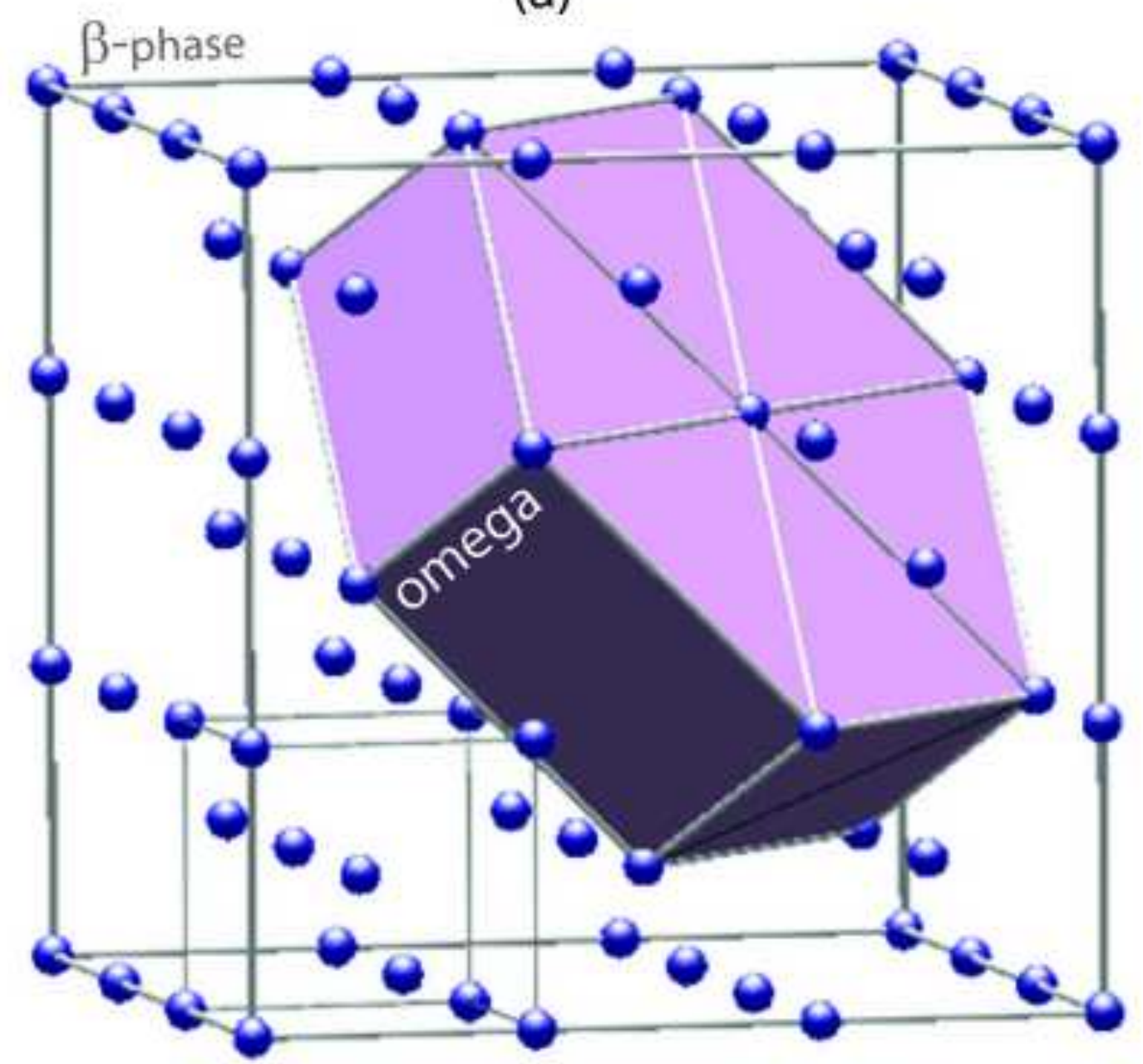

(b)

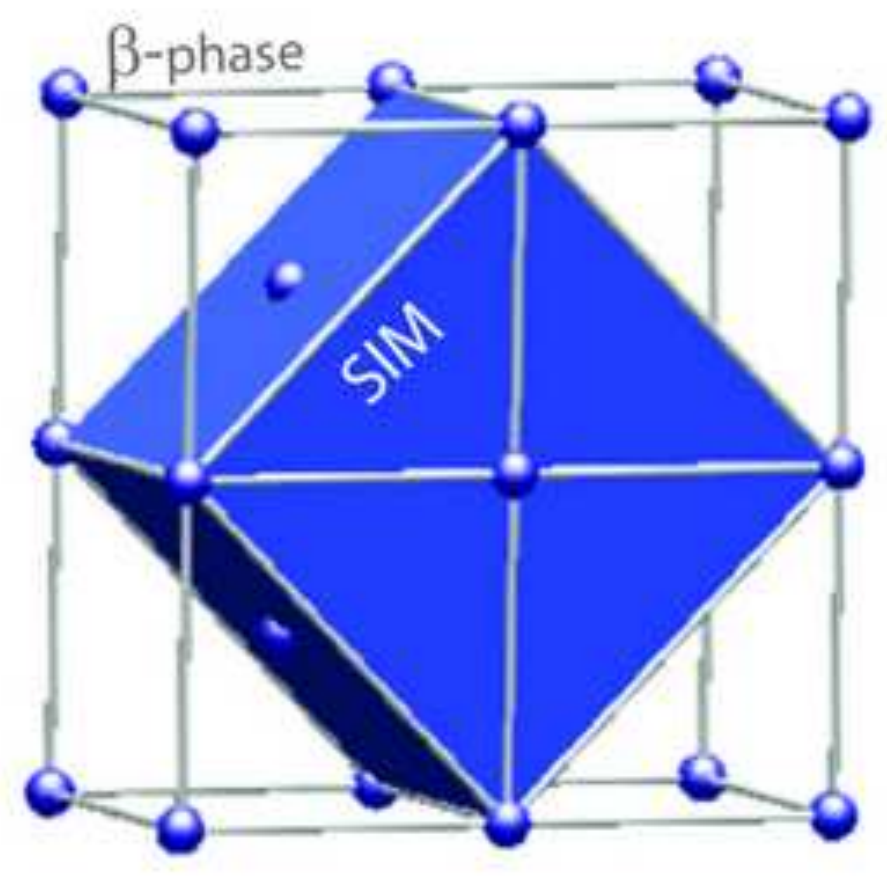


a) (310)
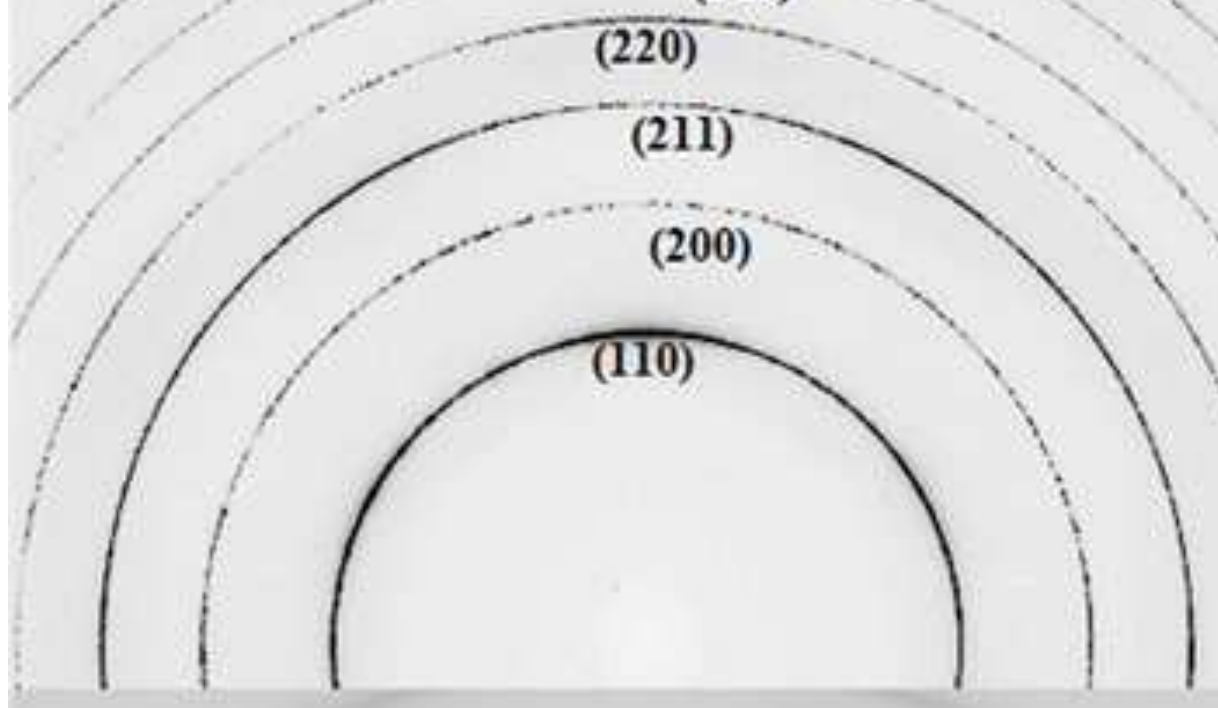

b)

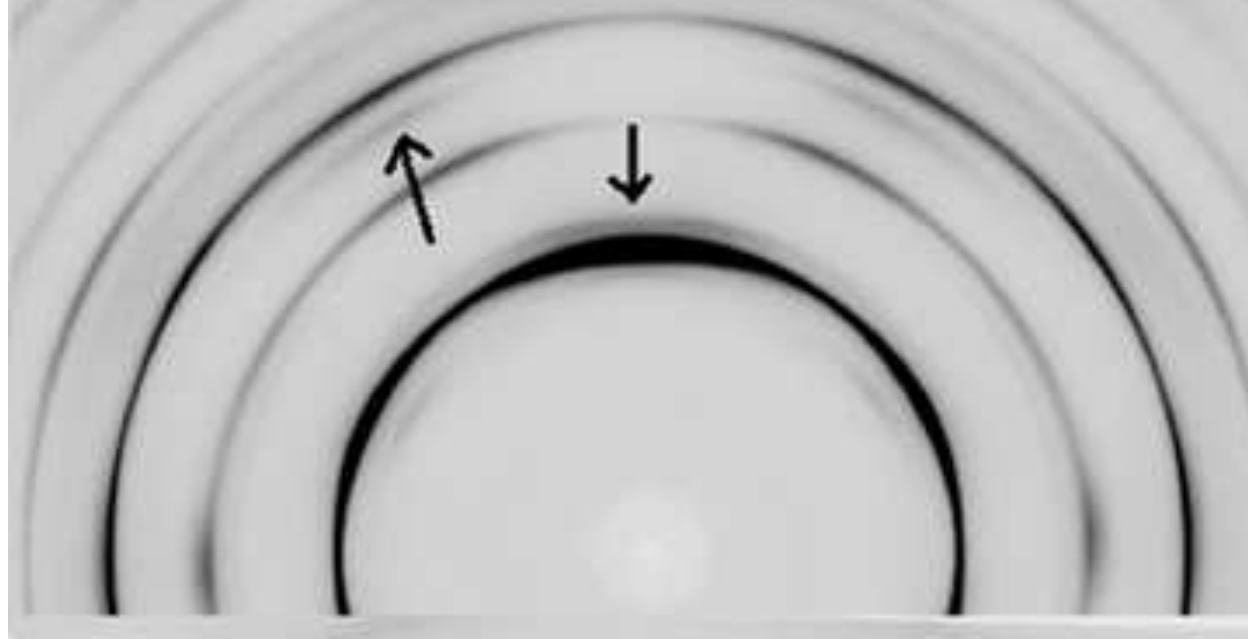

c) 


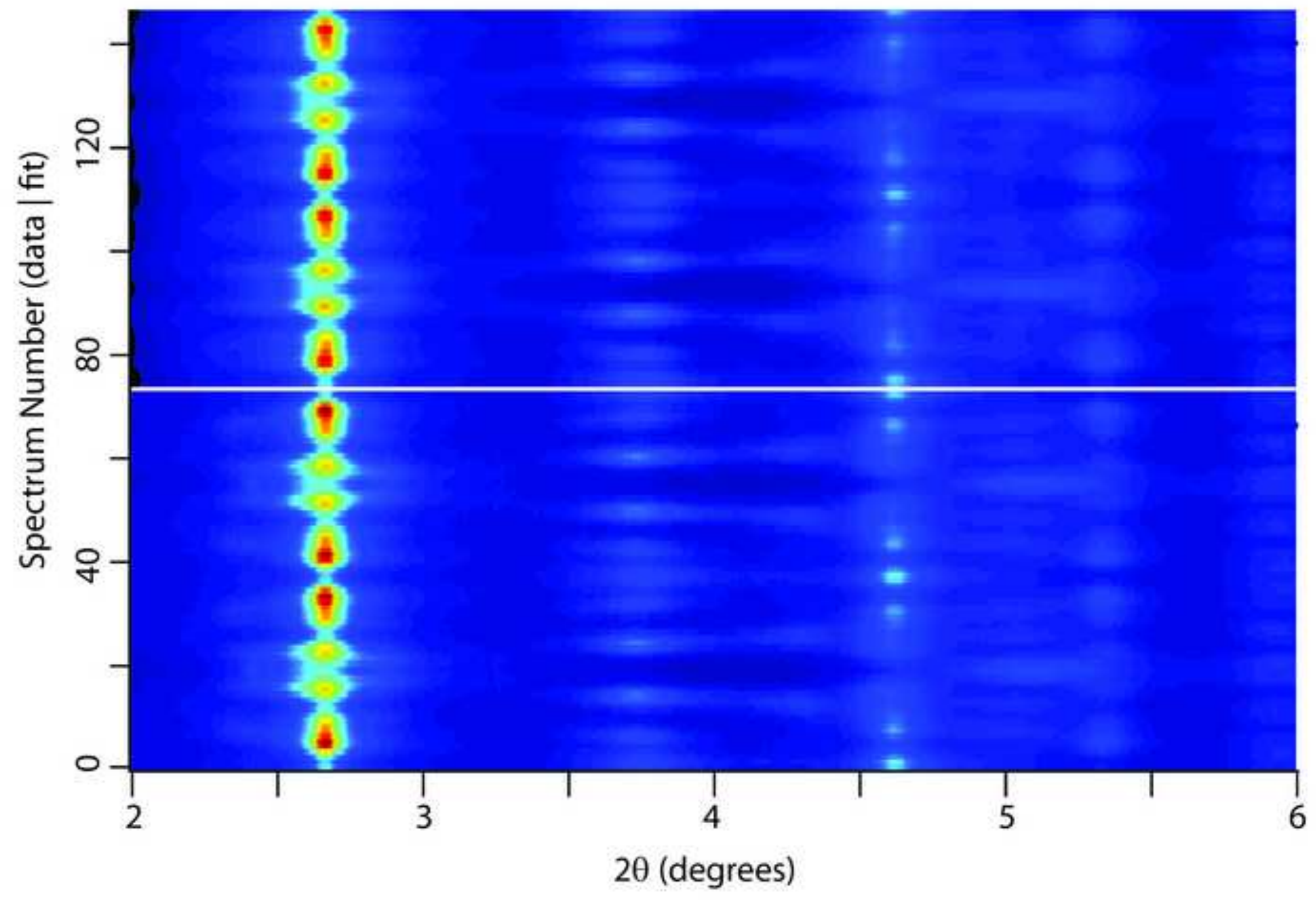




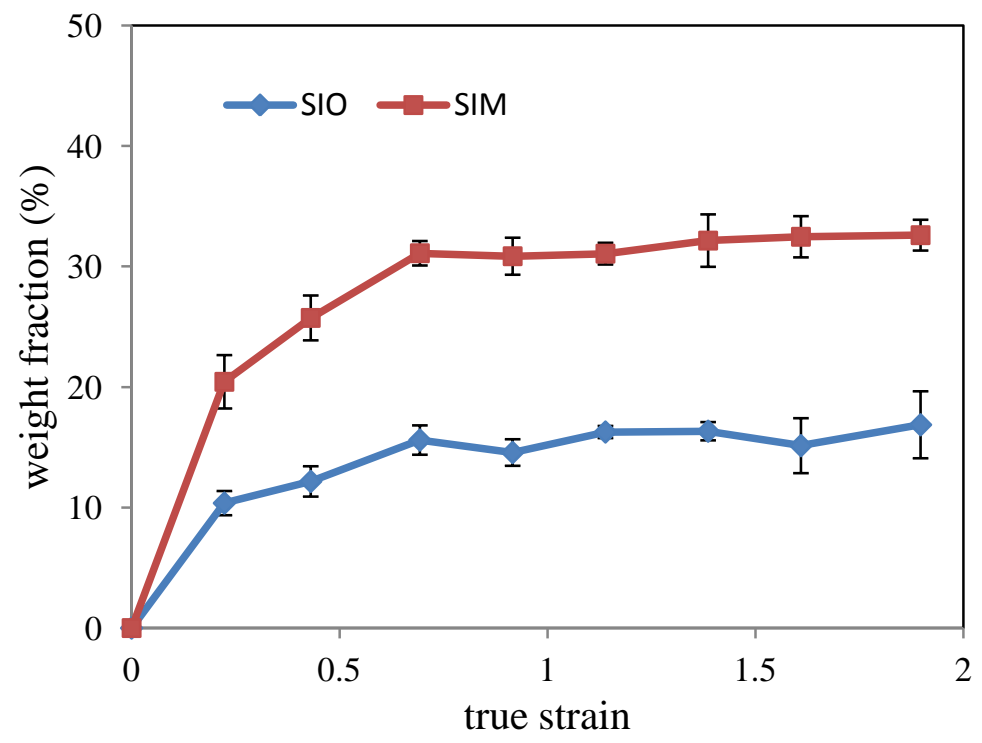



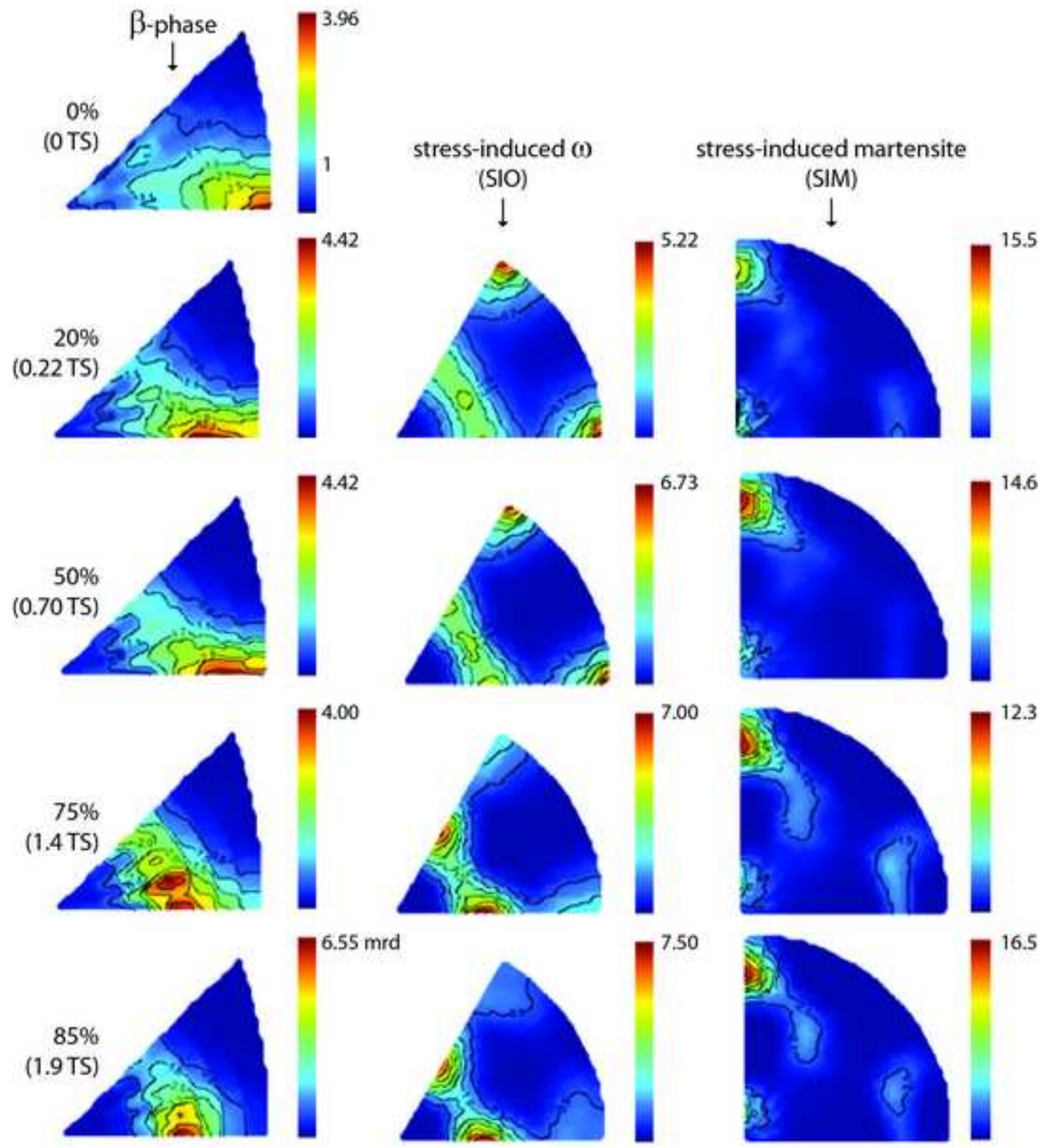
(110)

(200)

(222)

Cold work
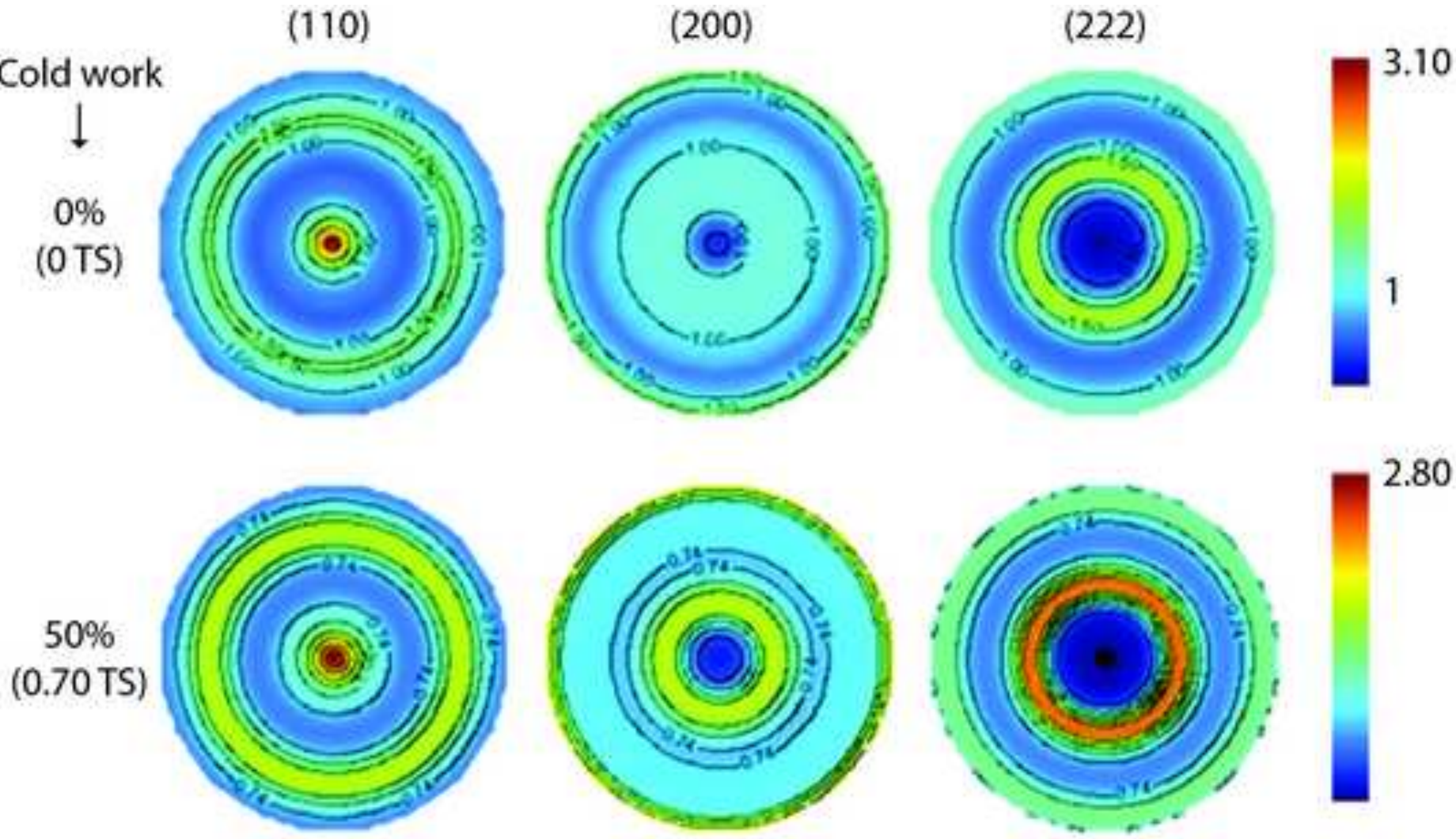

2.80
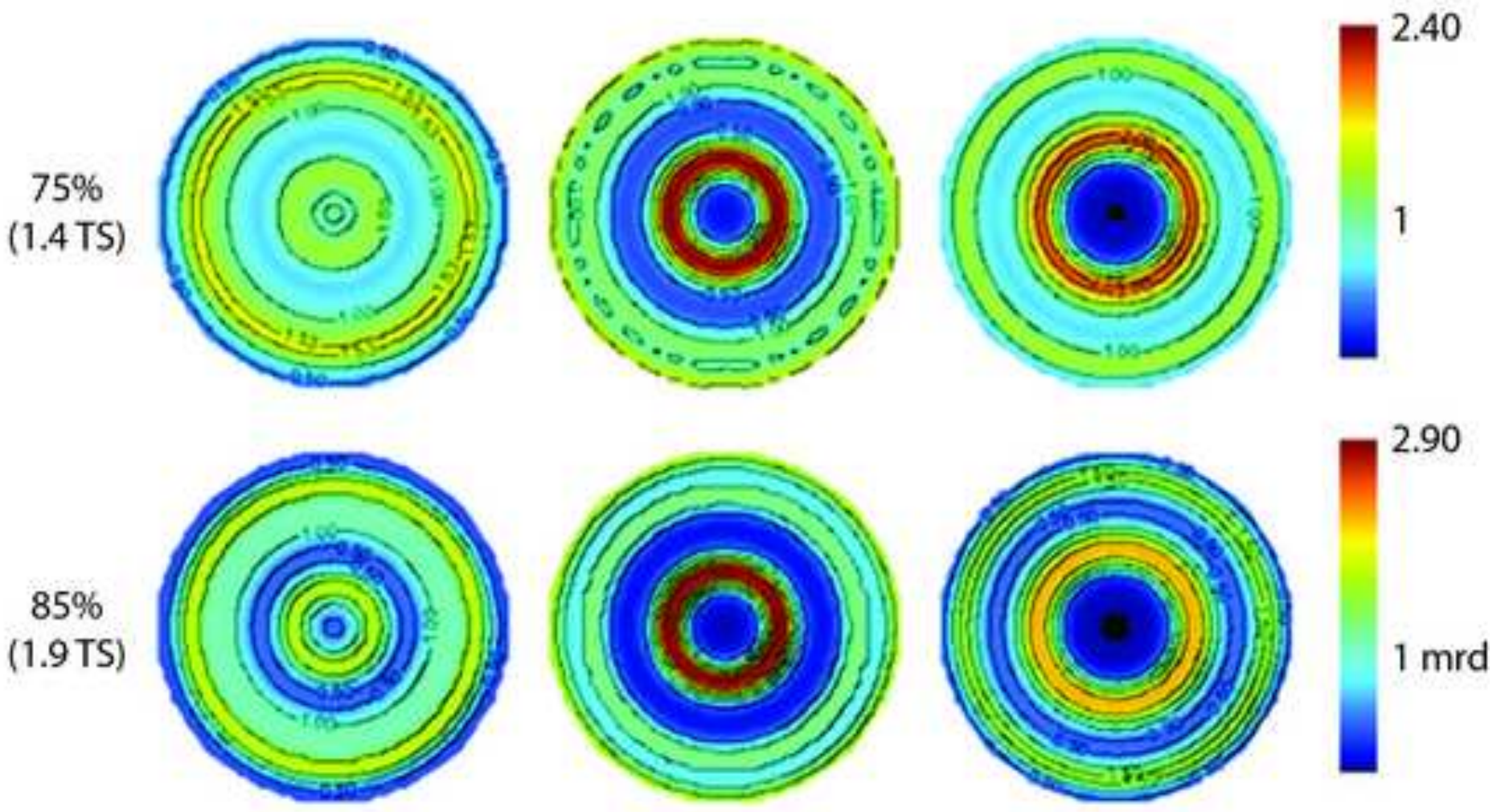

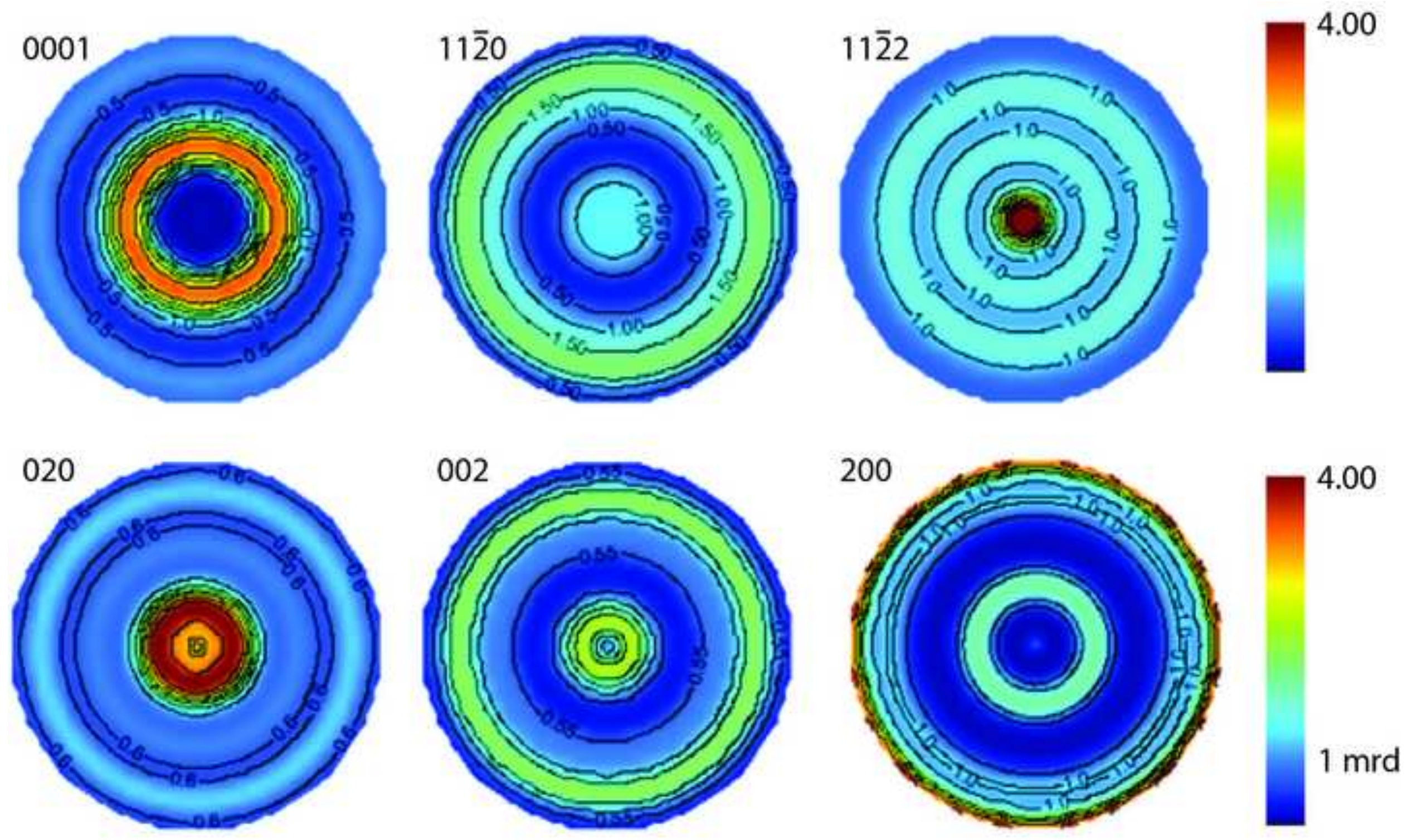


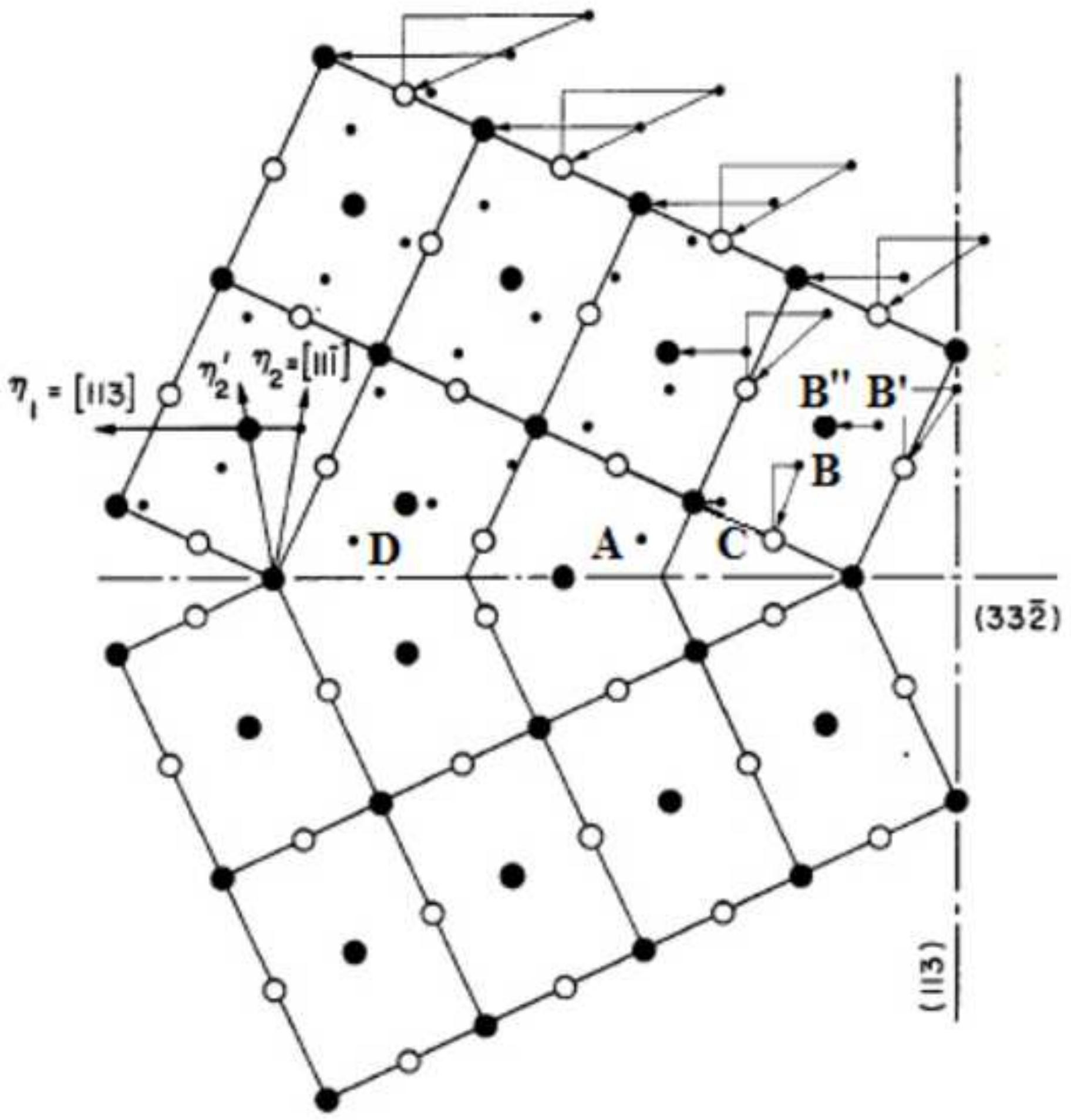


(110)
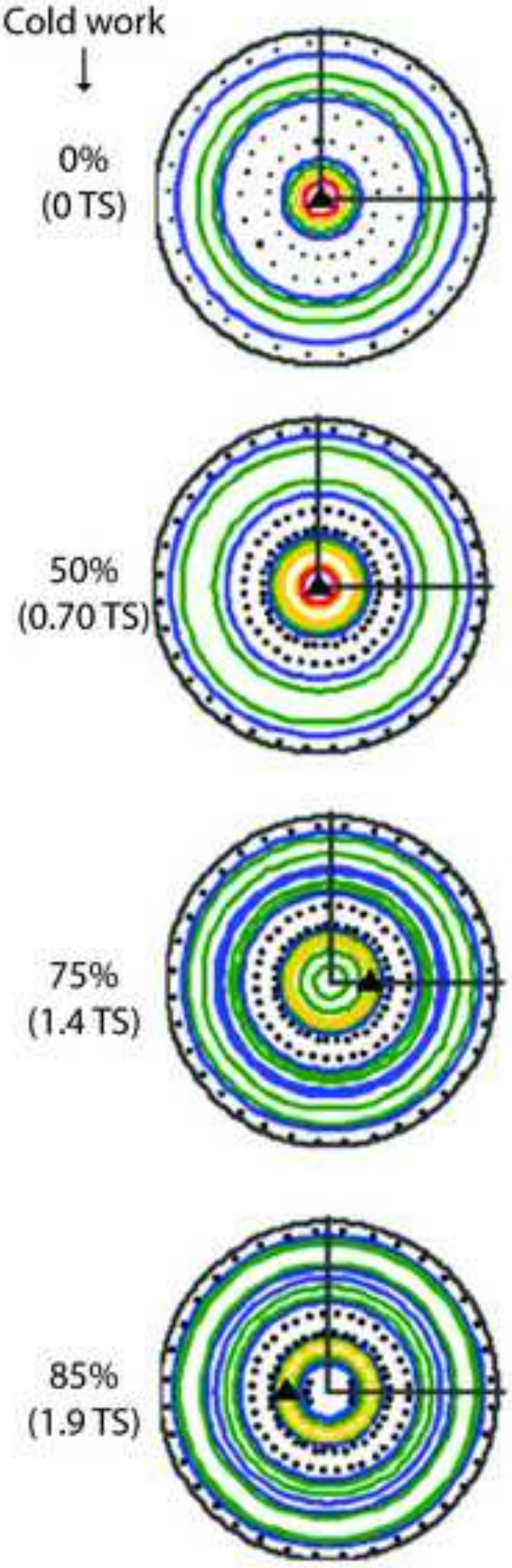

(200)
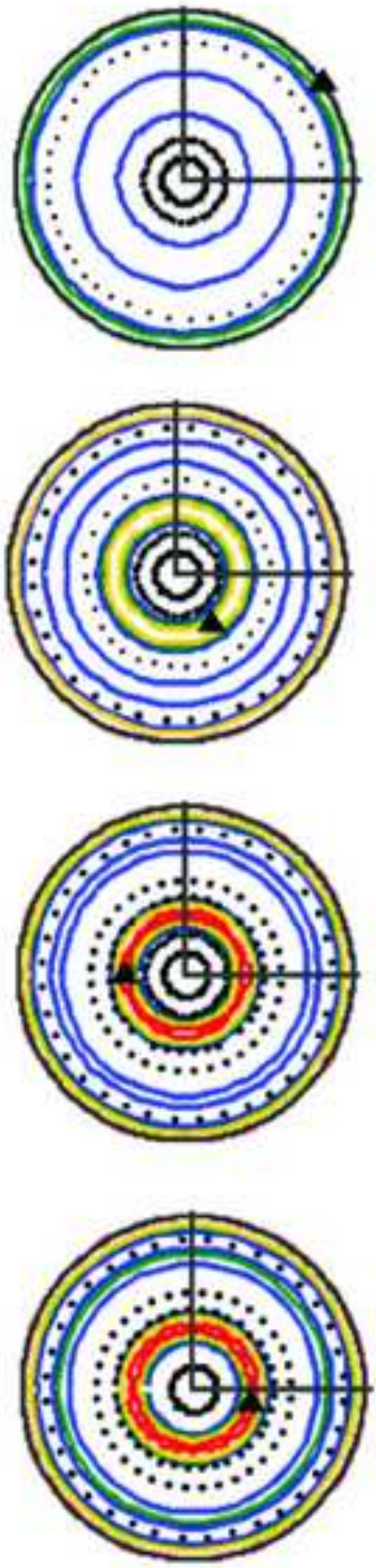

(222)
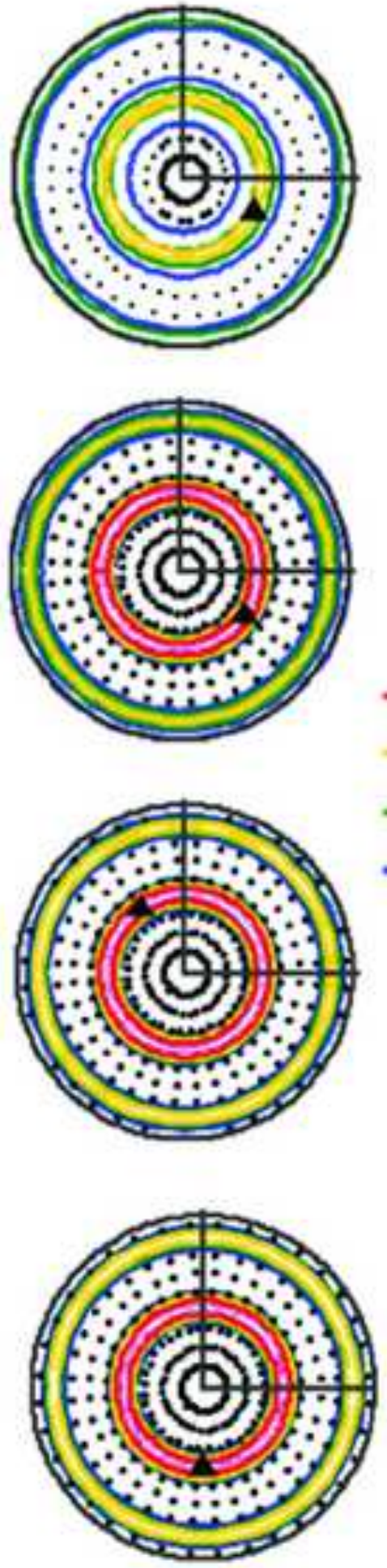

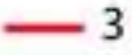

$-1 \mathrm{mrd}$ 


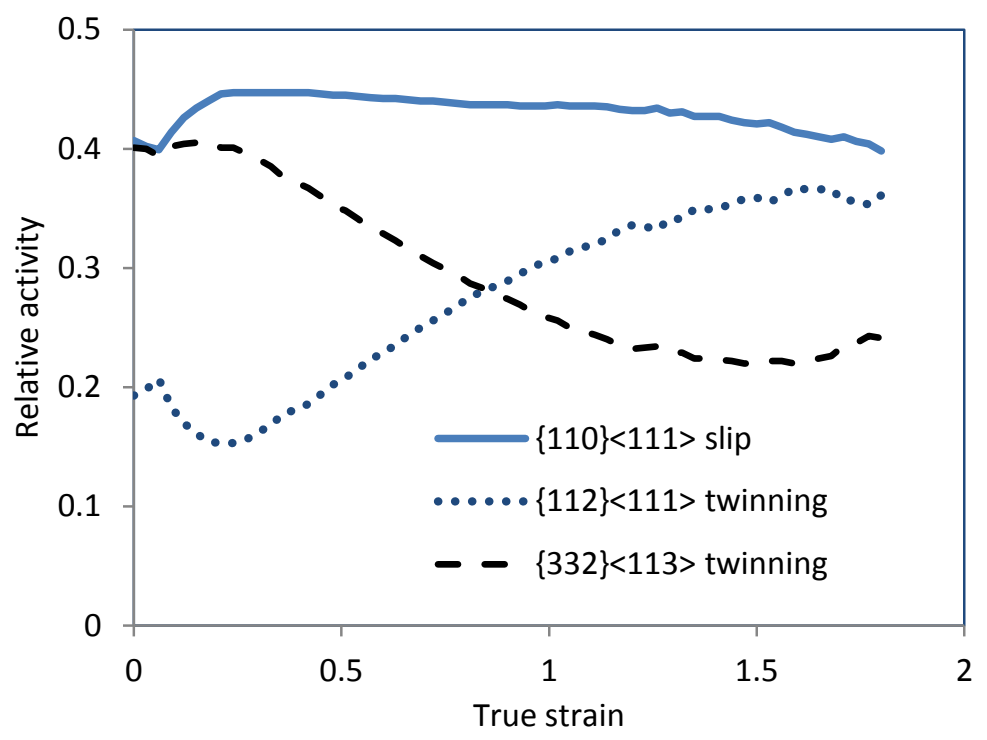




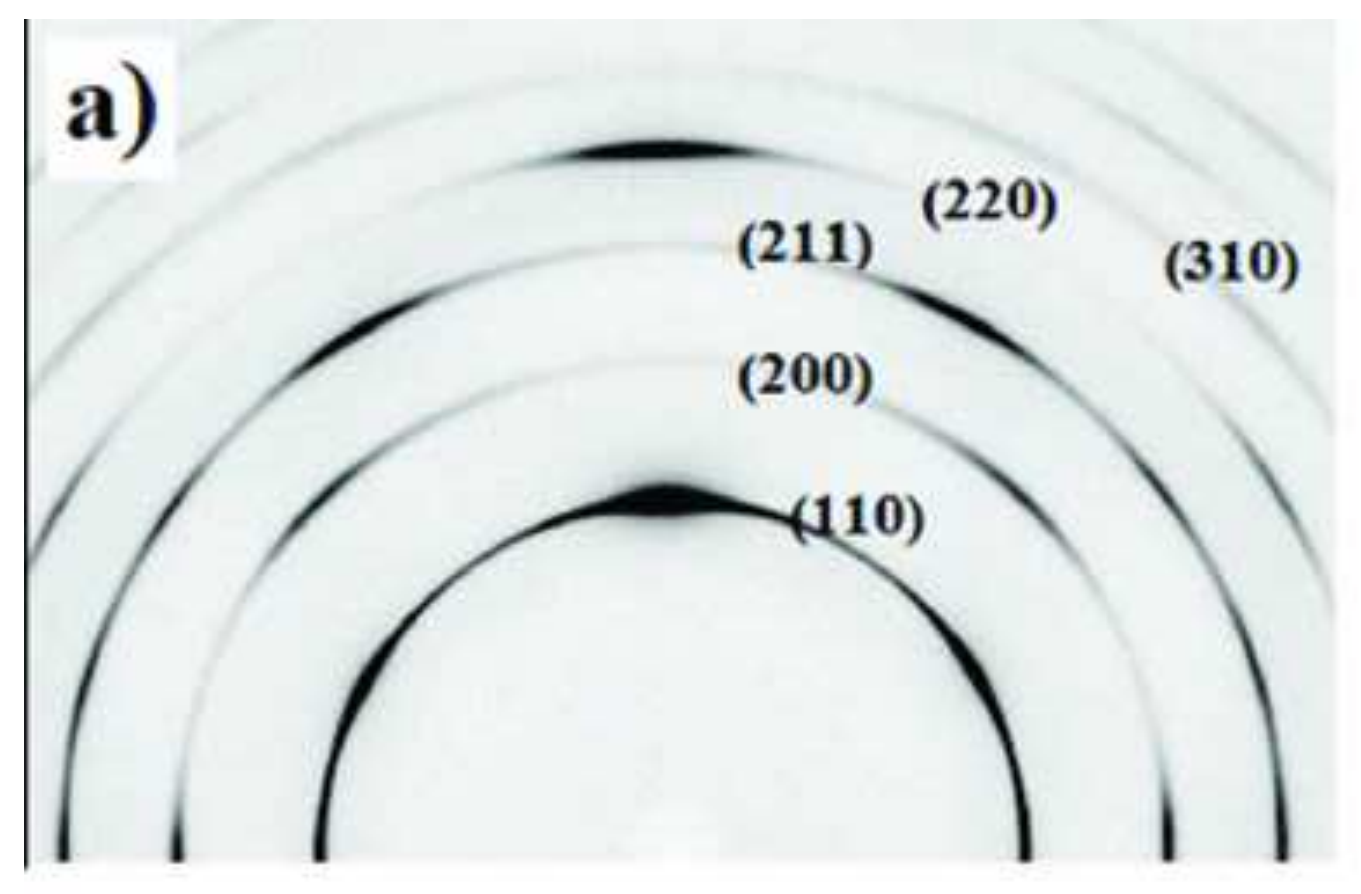

b)

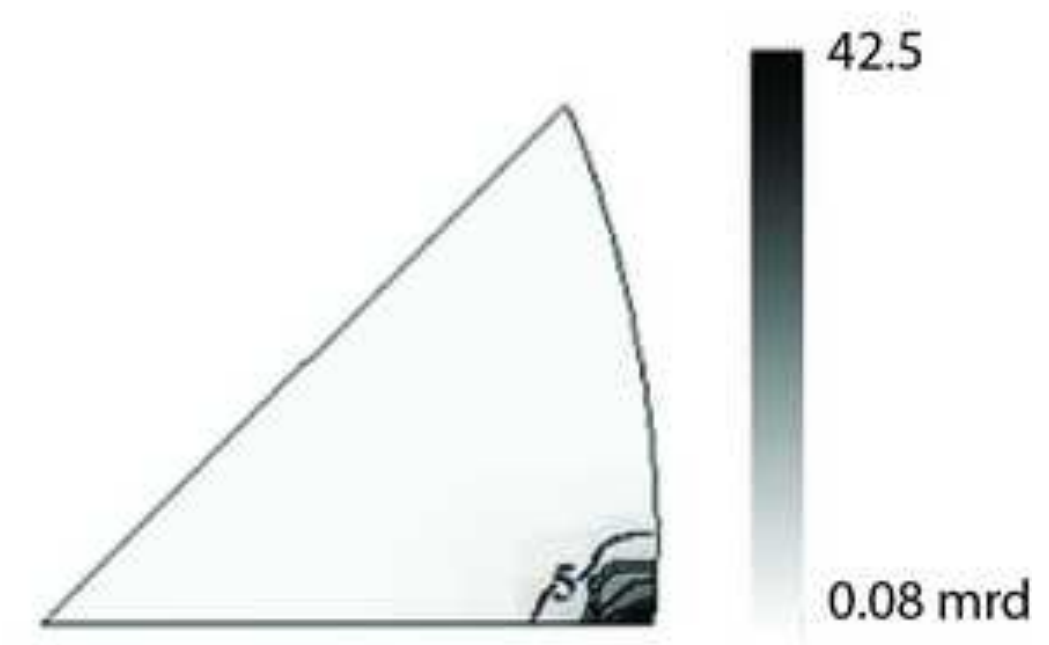

